\title{
Dream-land with Classic Higgs field, Dimensional Reduction and all that 1
}

\author{
D. V. Shirkov \\ Bogoliubov Lab., JINR, Dubna
}

Instead of Abstract

This text, on the one hand, is related to the talk delivered at the Conference "Gauge Fields. Yesterday, Today, Tomorrow", dedicated to the Andrej Slavnov 70th anniversary; on the other - in the form of a fairy tale - it summarizes some results of researches performed after this Fest, mainly due to discussion around the talk.

\section{Introduction}

\subsection{Motivation}

Initial impetus for treating the topic mentioned in the title is related to the hot quest of the Higgs particle still escaping from observation. Anticipating the case with no Higgs peak detected at all, we turn to possibility that Higgs is a classical field (being an analog of Ginzburg-Landau order parameter in the theory of superconductivity) with nonzero constant component sufficient for the mass production in the current version of SM.

However, changing a quantum Higgs for the classical external field yields the renormalization trouble in the EW sector of SM. Not entering this problem we looked for temporary practical remedy. The possible way is to use an invariant regularization procedure. To this goal, we exploit a transition from the four-dim space-time manifold to the one with a smaller number of dimensions $D=1+d<4$ at high enough energy. Technically, this provides us with one additional parameter, artificial cutoff scale or the range of reduction. The trick with changing the number of dimensions is a frequent one in literature (on superstrings etc.) devoted to the HE behavior. This Kaluza-Klein scenario with larger number of dimensions $D>4$ confronts us with the non-renormalizability. Instead, we consider another, an opposite possibility.

In Section 2, we use a $g \varphi^{4}$ QFT model with running coupling defined in both the two domains of $D=4$ and $D=2$; the $\bar{g}\left(Q^{2}\right)$ evolutions being duly conjugated at the reduction scale $Q \sim M=M_{d r}$. Beyond this scale, in deep UV 2-dim region, $\bar{g}$ does not increase any more and tends to a finite value $\bar{g}_{2}(\infty)<\bar{g}_{2}\left(M^{2}\right)$ from above. As a result, the global evolution picture looks quite peculiar and can provide a basis for the modified GUT scenario with dimensional reduction for unification instead of leptoquarks — see Fig. 8.

Then, Section 3 summarizes some very recent research that contains the study of Klein-Fock-Gordon eq. on toy examples with variable 2-dim space geometry including

\footnotetext{
${ }^{1}$ D. V. Shirkov, "Dreamland with classic Higgs field, dimensional reduction and all that", Proc. of the Steklov Inst. of Math., 2011, v. 272, pp.216-222
} 
reduction of the space dimension number from $d=2$ to $d=1$. Here, the specific trick of transforming the Klein-Fock-Gordon problem on variable geometry to Schrödinger-type eq. with potential generated by space variation was used.

The final Section 4 contains a short summary and outlook. For a detailed exposition we address to fresh publications - [1] (on Section 2) and [2, 3] (on Section 3).

\subsection{Dimensional reduction}

The diminishing of dimension number, dimensional reduction $(=\mathrm{DR})$ was used about 15 years ago (Aref'eva [4], and others) in the HE scattering context. More recently, it got a second wind in the quantum gravity.

To explore some practical aspects of the DR, we turn here to a limited subject, the transferring the renorm-invariant running coupling $\bar{g}\left(q^{2}\right)$ through the region of reduction and relating its behavior in two domains with different dimensionality.

In performing the DR procedure in the Lagrangian approach and in the energymomentum integration of Feynman integral we use assumption

DR Agreement. Reduction at the space-time scale $x_{d r} \sim 1 / M_{d r}$ is, in a sense, equivalent to reduction at the energy-momentum scale $p_{d r} \sim M_{d r}$.

In the course of the first approach we use a sharp conjunction as an approximation to a softer mechanism of a continuous DR in the second one.

Classical illustration. To illustrate the idea of approximation, take an empty wine bottle posed vertically like one presented on Fig. 1(a). It has a main cylindrical body with

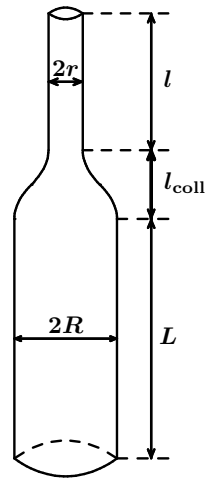

(a)

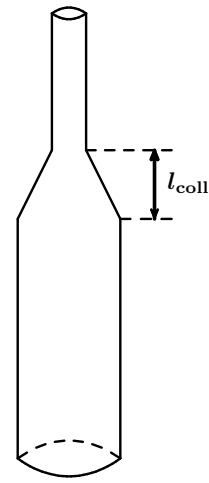

(b)

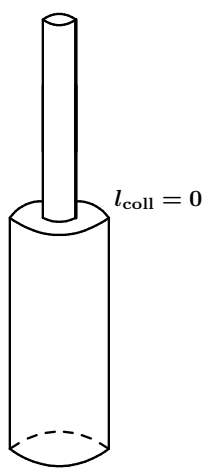

(c)

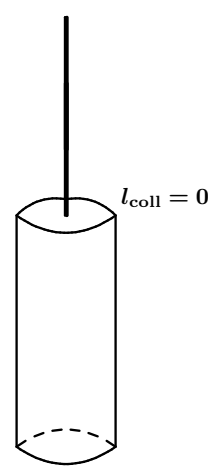

(d)

Figure 1: (a) - Wine-bottle with a long body and a long neck; (b) - Bottle with a conical collar: (c) - Bottle with a horizontal collar: (d) - Bottle with a horizontal collar and a thread-like neck.

a relatively large radius $R$ and a length $L$. The bottle's neck of length $l$ and smaller radius $r$ is connected with the main part by a "collar" - a transition region $C$ of varying radius and a short length $l_{\text {coll }}$.

Imagine now an equation(s) describing some process on the 2-dim surface $S_{2}=S_{R, L}+$ $S_{r, l}+S_{\text {coll }}$ of the bottle. One can mean a stationary boundary value problem, some dynamical process like wave propagation (with radiation) or heat conductivity and so 
on. A number of problems with exact analytical solutions on the surfaces $S_{R, L}$ and $S_{r, l}$ of cylindrical parts can be found. Many of them could be solved for the whole twodimensional manifold for simple enough forms of the junction collar region $C$.

In the further analysis, along with smooth transition in Section 2.1, we shall use "hard conjunction" of two regions with different dimensions. The first one is an analog of Fig. 1(a) in the limit $r \rightarrow 0$, while the second resembles the Fig. 1(d).

\section{Effective $\bar{g}$ for the $\varphi^{4}$ model in various dimensions}

Take the one-component scalar massive quantum field $\varphi(x)$ with the self-interaction

$$
L=T-V ; \quad V(m, g ; \varphi)=\frac{m^{2}}{2} \varphi^{2}+\frac{4 \pi^{d / 2}}{9} g \varphi^{4} ; \quad g>0
$$

in parallel in four $(D=4)$ and two $(D=2)$ dimensions.

Limit ourselves to the one-loop approximation level for $\bar{g}$ that corresponds to the only Feynman diagram contribution, the first correction to the 4-vertex function, Fig. 2.

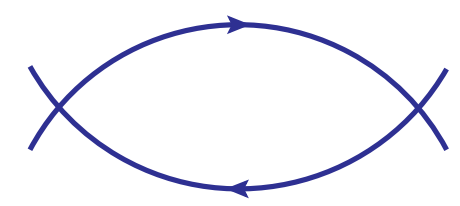

Figure 2: One-loop vertex diagram

Its subtracted contribution $I$ enters the effective running coupling as a whole :

$$
\bar{g}\left(q^{2}\right)=\frac{g_{i}}{1-g_{i} I\left(q^{2} ; m^{2}, m_{i}^{2}\right)} .
$$

\subsection{Smooth DR in the momentum picture}

For DR in momentum representation we modify the metric

$$
d k=d^{4} k \rightarrow d_{M} k=\frac{d^{4} k}{1+k^{2} / M^{2}} ; \quad k^{2}=\mathbf{k}^{2}-k_{0}^{2} .
$$

in the one-loop Feynman integrand Fig. 2

$$
I\left(\frac{q^{2}}{m^{2}}\right) \rightarrow \frac{i}{\pi^{2}} \int \frac{d_{M} k}{\left(m^{2}+k^{2}\right)\left[m^{2}+(k+q)^{2}\right]}=J(\kappa ; \mu) ; \quad q^{2}=\mathbf{q}^{2}-q_{0}^{2} ; \kappa=\frac{q^{2}}{4 m^{2}} ; \mu=\frac{M^{2}}{m^{2}} .
$$

Turn to UV asymptotics. In the "deep 4-dim" region $m^{2} \ll q^{2} \ll M^{2}$ one gets an "intermediate" logarithmic behavior with the $M$ playing the role of the Pauli-Villars regulator. Meanwhile, in the "deep 2-dim" region $q^{2} \gg M^{2} \gg m^{2}$, the UV limit is finite. In usual normalization

$$
J \rightarrow J_{i}=J\left(q^{2} / 4 m^{2} ; \mu\right)-J\left(m_{i}^{2} / 4 m^{2} ; \mu\right) ; \quad m_{i} \sim m
$$


one has

$$
J_{i}^{[4]}(\kappa ; \mu) \sim \ln \left(\frac{q^{2}}{m_{i}^{2}}\right) ; \quad J_{i}^{[2]}(\kappa ; \mu) \sim \ln \left(\frac{4 M^{2}}{m_{i}^{2}}\right)+\frac{M^{2}}{q^{2}} \ln \frac{q^{2}}{M^{2}} .
$$

The first expression is rising; while the second, decreasing. The maximum value of $J$ is attained at the DR scale $q^{2} \sim M^{2}$ and is close to $\ln \mu$. Hence, due to the DR, the evolution of the coupling $\bar{g}\left(q^{2}\right)$ changes; it diminishes beyond the reduction scale and tends to a finite value as in Fig. 3.

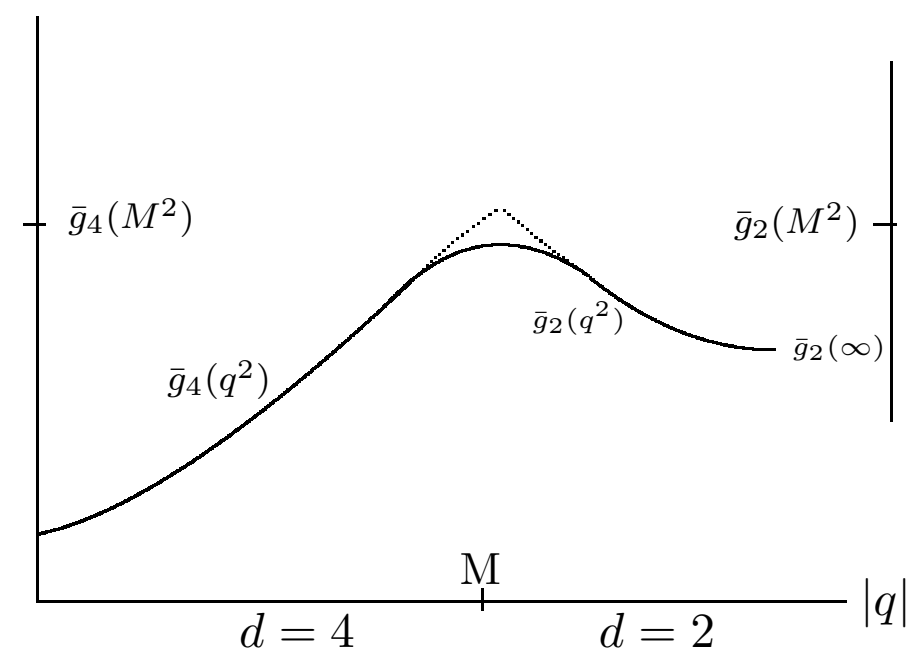

Figure 3: The effective coupling evolution for the $\varphi^{4}$ model with DR.

\section{$2.2 \quad$ Reduction with Lagrangian}

Now, along with the DR Agreement, turn to the Lagrangian description eq.(1). There, under transition to the $D=2$ case, the scalar field loses its dimensionality $\varphi_{4}(x) \rightarrow$ $\varphi_{2}(x) \sim M \varphi_{4}(x)$, while the coupling constant acquires it: $g_{4} \rightarrow g_{2} \sim M^{-2} g_{4}$ with one parameter that can be put equal to the DR scale $M=M_{d r}$.

Invariant coupling in $d=2$. In two dimensions, one can use finite Dyson transformations and formulate mass-dependent renorm-group (as it was first introduced [5] in the mid-50s)

$$
\bar{g}^{[2]}\left(q^{2}\right)=\frac{g}{1-g I_{2}\left(q^{2} / m^{2}\right)} ; \quad I_{2}\left(\frac{q^{2}}{m^{2}}\right)=\frac{i}{\pi} \int \frac{d^{2} k}{\left(m^{2}+k^{2}\right)\left[m^{2}+(k+q)^{2}\right]} .
$$

Here, $I_{2}$ is a finite contribution from 4 -vertex diagram, Fig. 2. It is a positive monotonously decreasing function. Asymptotically, $I_{2} \sim \ln \left(q^{2} / m^{2}\right) / q^{2}$, as in the second eq.(3). Hence, two-dim coupling in the UV limit tends to its limiting fixed value from above.

Hard conjunction at the reduction scale. To obtain the joint picture of coupling evolution, consider transition from the "low-energy" 4-dim region $q^{2}<M^{2}$ to "high-energy" 
2-dim one $q^{2}>M^{2}$. For the "hard" conjunction (analog of Fig. 1(d)) the continuity condition $\bar{g}_{4}\left(M^{2}\right)=\bar{g}_{2}\left(M^{2}\right)=g_{M}$ yields the finite UV limit

$$
\bar{g}_{2}(\infty)=\frac{g_{M}}{1+g_{M} I_{2}\left(M^{2} / m^{2}\right)}<g_{M} .
$$

This means that above the reduction scale the effective coupling evolves from $g_{M}$ down to $\bar{g}_{2}(\infty)$ that corresponds to Fig. 3 .

\section{Klein-Fock-Gordon equation on variable geometry}

Our attitude does not imply any modification of the concept of time. Instead, we have in mind some continuous transformation of spatial geometry up to reduction of space dimensions.

To get physical intuition and experience, we start with Klein-Fock-Gordon scalar waves[2] on some toy models of space (like on Fig. 1) with variable geometry.

Klein-Fock-Gordon equation (KFGEq) on a composite space can be presented in the form

$$
\square \varphi-M^{2} \varphi=0 ; \quad \square=-\frac{1}{\sqrt{|g|}} \partial_{\mu}\left(\sqrt{|g|} g^{\mu \nu} \partial_{\nu}\right)=-\partial_{t t}^{2}+\Delta_{d},
$$

with $d$-dim operator $\Delta_{d}$ on the $d$-dim metric $\gamma_{m n}$

$$
\Delta_{d}=\frac{1}{\sqrt{|\gamma|}} \partial_{m}\left(\sqrt{|\gamma|} \gamma^{m n} \partial_{n}\right), \quad m, n=1, \ldots, d
$$

At the $(1+d)$-formalism with common time any global solution $\varphi$ on the parts with different dimension $d_{1}, d_{2}, \ldots$ have a common frequency $\omega=\omega_{1}=\omega_{2}=\ldots$.

On the cylinder-symmetry $2 \mathrm{~d}$-surface with shape function $\rho(z)$

$$
(d l)^{2}=\gamma_{m n} d x^{m} d x^{n}=\rho^{2}(z) d \phi^{2}+\left(1+\rho^{\prime 2}\right) d z^{2},
$$

the Laplacian is $\Delta_{2}=\frac{1}{\rho^{2}}\left(\partial_{\phi \phi}^{2}+\frac{\rho}{\sqrt{1+\rho^{\prime 2}}} \partial_{z} \frac{\rho}{\sqrt{1+\rho^{\prime 2}}} \partial_{z}\right)$. Separating variables $\varphi(t, \phi, z)=$ $=T(t) \Phi(\phi) Z(z)$ one gets two simple equations $T^{\prime \prime}+\omega^{2} T(t)=0 ; \quad \Phi^{\prime \prime}+m^{2} \Phi(\phi)=0$, and the more involved Z-equation

$$
\frac{1}{\rho \sqrt{1+\rho^{\prime 2}}} \partial_{z}\left(\frac{\rho}{\sqrt{1+\rho^{\prime 2}}} \partial_{z} Z\right)+\left(\omega^{2}-M^{2}-\frac{m^{2}}{\rho(z)^{2}}\right) Z(z)=0,
$$

with centrifugal potential $\mathrm{m}^{2} / \rho(z)^{2}$ due to motion in curved space.

Our next result can be formulated as "Dynamics instead of Geometry". Indeed, Eq.(Z) can be transformed to a proper Schrödinger-like eq. :

$$
\psi^{\prime \prime}(u)+(E-V(u)) \psi(u)=0
$$

by change of coordinate and shape function

$$
z \mapsto u: \quad u(z)=\int \sqrt{1+\rho^{\prime}(z)^{2}} \frac{d z}{\rho(z)} ; \quad \rho(z) \rightarrow \varrho(u)=\rho(z(u)) .
$$




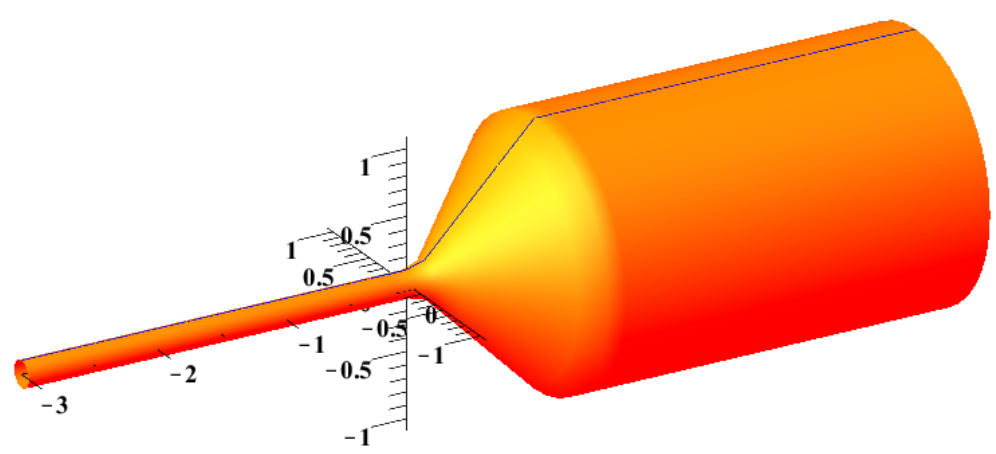

Figure 4: The first toy model.

Then $\Delta_{2}=\varrho^{-2}\left(\partial_{\phi \phi}^{2}+\partial_{u u}^{2}\right)$ with $\varrho(u)^{2}$ being 2-dim conformal factor, and

$$
E=0, \quad V(u)=\left(M^{2}-\omega^{2}\right) \varrho(u)^{2}+m^{2}, \quad Z(z)=\psi(u(z)) .
$$

Thus, study of KFGEq on a curved manifold is reducible to the Schrödinger-like eq. solving (with potential $V(u)$ defined by geometry).

Consider now KFGEq on surface of two cylinders connected by part of cone with shape function (Fig.4)

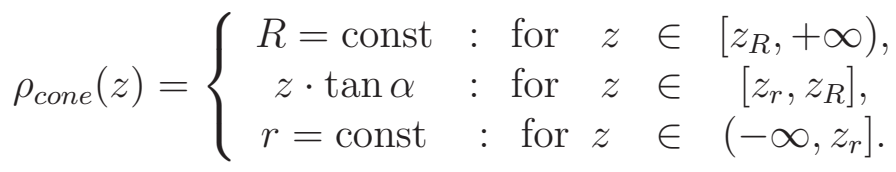

In the (singular) limit $r \rightarrow 0$ the model describes dimension reduction $d=2 \rightarrow 1$. It admits analytic solution in terms of Bessel functions and getting spectra. The related spectra depend on the KFG mass M, as shown on Fig.5.
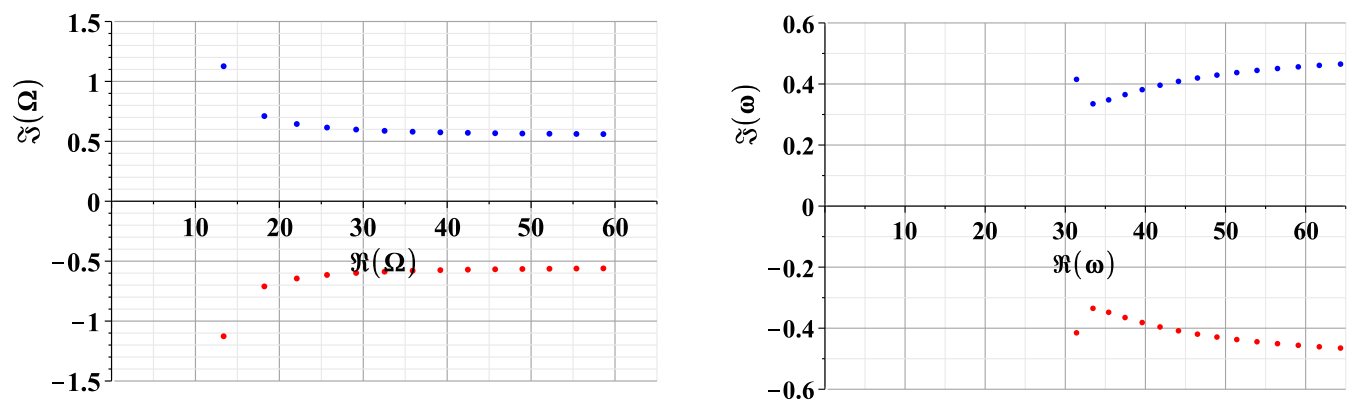

Figure 5: Complex spectra $\omega_{n, m}=R e \omega_{n, m}+i \operatorname{Im} \omega_{n, m}, n=0,1,2$, for $m=10, \alpha=\pi / 3$ and masses $M=0$ (left panel) and $M=28.85$ (right panel) The sequence of points with $\operatorname{Im} \omega_{n, m}>0$ corresponds to particles. The sequence of points with $\operatorname{Im} \omega_{n, m}<0$ corresponds to antiparticles. 
Another analytically soluble case refers to conic-like (with angle $\alpha$ at the top of the cone) junction with thin cylinder and with smooth transition (ST) to big cylinder (Fig.66). It allows to find solutions for class of Smooth Transition shapes $\rho_{S T}(z)$ and get spectra of KFG problem including limiting DR case $r=0$.

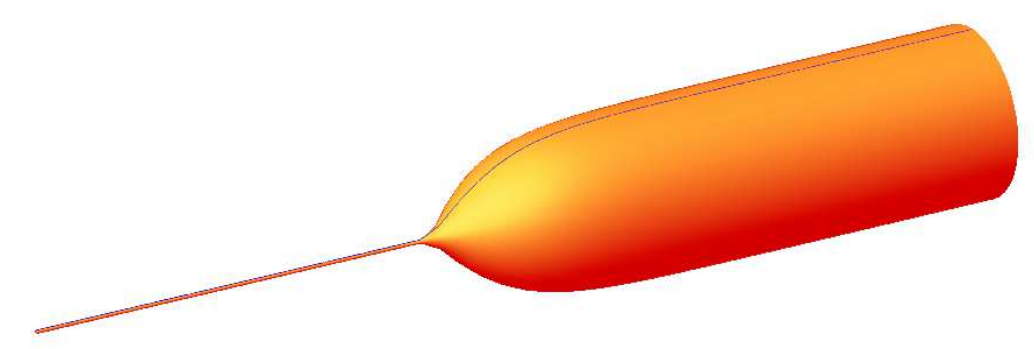

Figure 6: Second toy model with angle $\alpha$ at the apex of the cone.

The spectra for "2 Cylinders + smooth junction" (Fig. 7) contain two series2 2 of real frequencies $\omega_{n, m}$. We show spectra for two values of the mass $M=1,2$, the top angle value $\sin \alpha=0.2$ and angular momentum $m=2$.

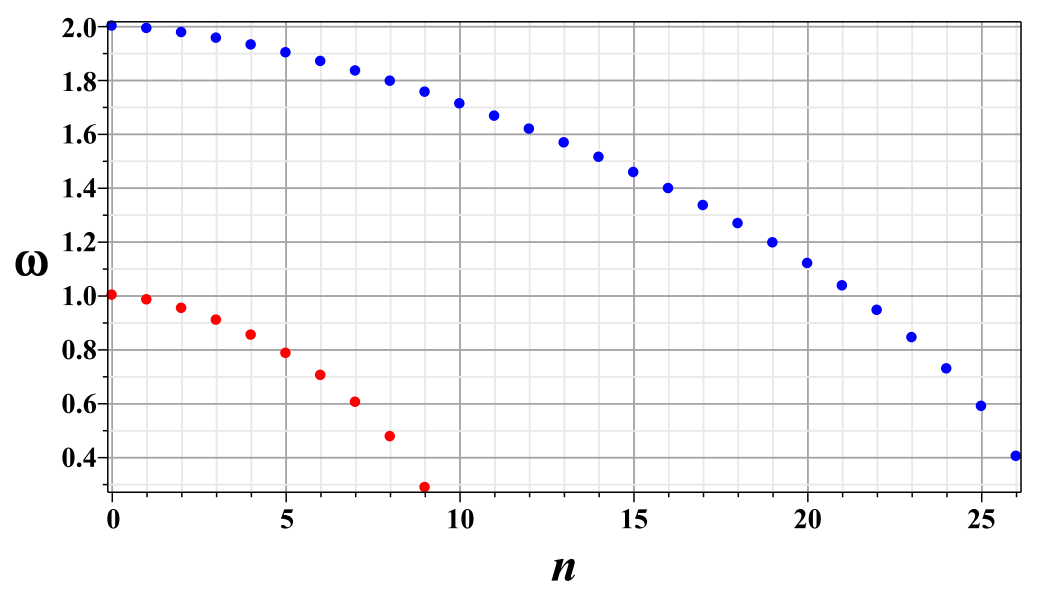

Figure 7: Real spectra for $\sin \alpha=0.2, m=2$. Ten red points correspond to $M=1$, whereas 27 blue points - to $M=2$.

Comparing spectra of $\rho_{\text {cone }}$ case with various $\rho_{S T}$ ones suggests a new idea: One can try to generate and explain observed spectra of particles using proper geometry of the junction between domains of space with different dimensions.

\footnotetext{
${ }^{2}$ It is curious that 'minus solutions' $\omega_{n, m}^{-}$are "mounted from the cellar". In usual treating they are unphysical and omitted in text-books.
} 


\section{Conclusion}

In the above analysis, one more alternative to the standard Higgs mechanism within the Standard Model was considered. The idea consists in employing the possibility of reducing the number of dimensions in the far UV limit.

First, taking ad hoc the reduction from four $(3+1)$ to two $(1+1)$ dimensions at some high enough scale $|q| \sim M_{d r}$, we studied the issue of effective coupling behavior for the $\varphi^{4}$ scalar model.

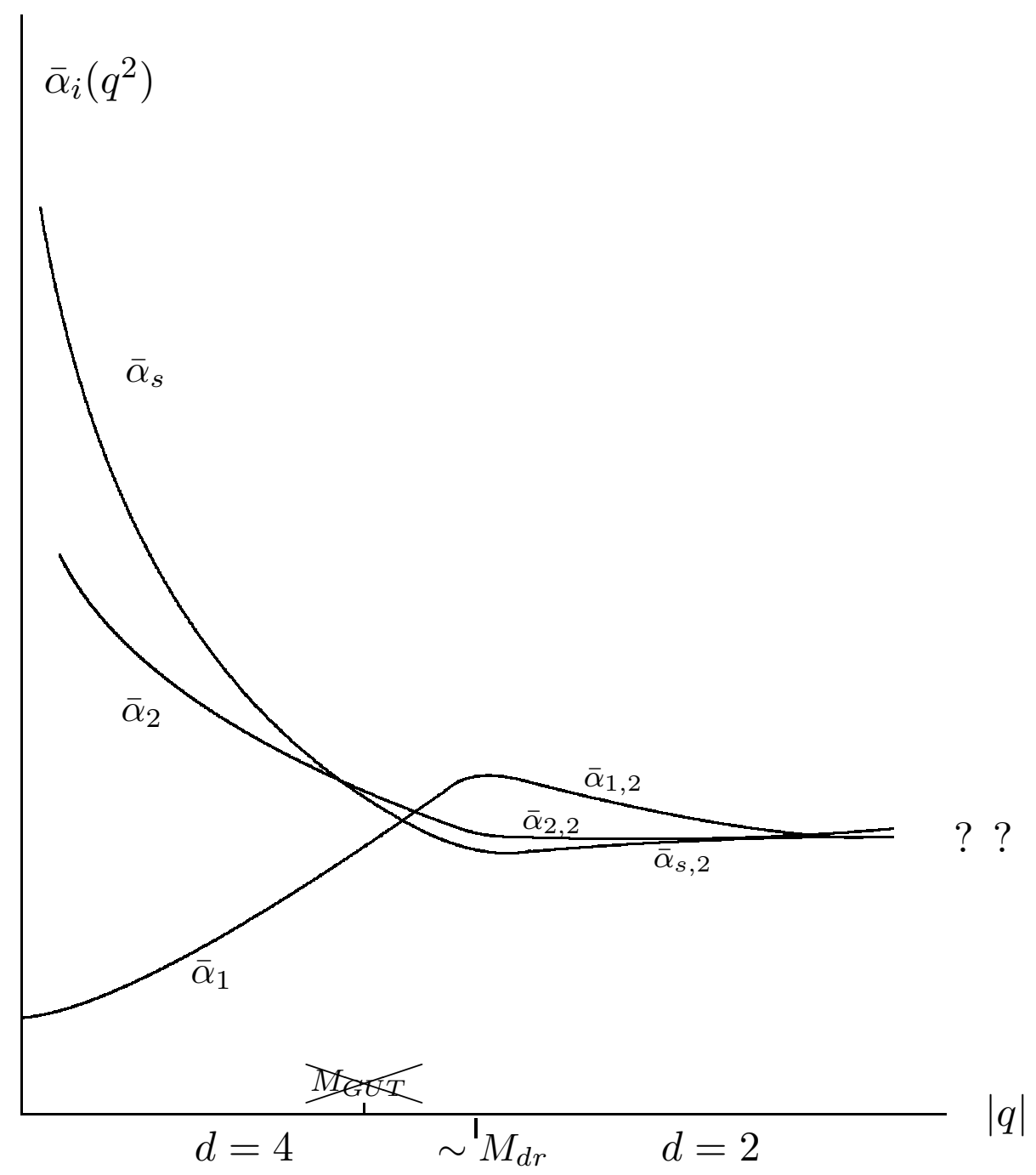

Figure 8: Modified chart of the GUT scenario provided by dimensional reduction instead of leptoquarks.

The peculiar property, the reverse running coupling evolution to a fixed point beyond the "distorted looking-glass" marked by the reduction scale has a chance to upgrade the Grand Unification scenario with the $M_{d r}$ value being of the order or even greater than the hypothetical lepto-quark scale — see Fig. 8. 
Second, we looked for the Klein-Fock-Gordon eq. solution on few toy models of space (like presented on Fig. 1) with variable geometry. Here, the results are

\section{A. On Signals between domains with Variable Geometry:}

a1. Communication between diverse dimension domains by wave signals with $m \neq 0$ is impossible.

a2. In the 2-dim domain for real frequency $\omega$ there is a total reflection on the cone of the wave coming from $z=+\infty$, (with change of the scattered wave phase).

\section{B. Novel Phenomena:}

The shape of effective mass and life-time excitation spectrum depends on the geometry of junction between different flat spaces.

Thus, spectrum is a specific "fingerprints" of junction region which characterizes its Geometry. Starting from spectrum one can re-construct the shape of junction. In acoustics this problem was posed by Lord Rayleigh (1877), then advanced by Hermann Weil (1911) and later by others.

\section{Acknowledgments}

It is a pleasure to thank Drs. Irina Aref'eva and Plamen Fiziev for numerous useful discussions. The courtesy of Dr. Jean Zinn-Justin for informing on relevant literature is also appreciated. This research has been partially supported by the presidential grant Scientific School 3810.2010.2 and by RFFI grants 08-01-00686, 11-01-00182

\section{References}

[1] D. Shirkov, Phys. Part. Nucl. Lett. v. 7: 379-383 (2010); arXiv:1004.1510[hep-th].

[2] Fiziev P. P. and Shirkov D. V., Theor. Math. Phys. v. 167: 680-691 (2011); arXiv:1009.5309v2 [hep-th].

[3] Fiziev P. P. and Shirkov D. V., J. Phys. A v. 45: 055205/1-15 (2012), doi:10.1088/1751-8113/45/5/055205; arXiv:1104.0903v2 [gr-qc].

[4] I. Ya. Aref'eva, Phys. Lett. B, v. 325: 171-182 (1994); arXiv:hep-th/9311115.

[5] N. N. Bogoliubov and D. V. Shirkov, Nuovo Cim., v. 3: 845-863 (1956). 